ISSN 1991-8631

Original Paper

http://indexmedicus.afro.who.int

\title{
Evaluation agro-morphologique des variétés et/ou écotypes locaux d'oignon du Niger
}

\author{
Habsatou BOUKARY ${ }^{1}$, Adamou HAOUGUI ${ }^{1}$, Moussa BARAGE ${ }^{2 *}$, Toudou ADAM ${ }^{2}$, \\ Albert ROUMBA ${ }^{3}$ et Mahamane SAADOU ${ }^{4}$ \\ ${ }^{1}$ Institut National de la Recherche Agricole du Niger (INRAN), BP. 429, Niamey, Niger. \\ ${ }^{2}$ Faculté d'Agronomie de l'Université Abdou Moumouni, BP. 10960, Niamey, Niger. \\ ${ }^{3}$ Vegetable Breeder, AVRDC -The World Vegetable Center Subregional Office for West and Central Africa, \\ BP 320, Bamako, Mali \& Institut d'Etudes et de Recherches Agricoles (INERA), BP. 8645, Ouagadougou, \\ Burkina Faso. \\ ${ }^{4}$ Université de Maradi et Faculté des Sciences de l'Université Abdou Moumouni, BP. 10662, Niamey, Niger. \\ *Auteur correspondant ; E-mail : moussa.barage@yahoo.fr
}

\section{RESUME}

Vingt et une (21) variétés et/ou écotypes locaux d'oignon collectés dans différentes régions du Niger ont été caractérisés dans la station expérimentale de l'INRAN à Konni dans le sud-est du Niger. Les caractères agronomiques qualitatifs et quantitatifs étudiés sont ceux recommandés par l'Institut International des Ressources Phytogénétiques (IPGRI, 2001). Ainsi, la longueur, la largeur, le poids, la couleur et la densité des feuilles, le port, la vigueur des plants, le poids, le diamètre, la hauteur, la couleur et l'uniformité des bulbes et le nombre de bourgeons végétatifs ont été observés. A l'issue de l'analyse des résultats, il ressort une corrélation positive entre la longueur des feuilles et la hauteur de bulbes $(0,704)$, le diamètre des bulbes et le rendement $\left(r^{2}=0,653\right)$, la hauteur et le diamètre des bulbes $\left(r^{2}=0,618\right)$, le nombre de bourgeons végétatifs et la matière sèche $\left(\mathrm{r}^{2}=0,570\right)$, et la longueur des feuilles et le cycle $\left(\mathrm{r}^{2}=0,522\right)$. Après analyse des caractères agro-morphologiques et physiologiques quantitatifs, trois groupes ont été remarqués dont $71 \%$ de variétés et/ou écotypes locaux pour le premier, $19 \%$ pour le deuxième et $10 \%$ pour le troisième, tandis qu' aucune corrélation n'a été observée au niveau des caractères qualitatifs. La variabilité observée dans les groupes est plus grande que celle qui existe entre les groupes, ce qui explique la présence des écotypes de différentes couleurs et différentes régions dans un même groupe. Une analyse moléculaire est plus que nécessaire pour confirmer cette diversité des variétés et/ou écotypes locaux d'oignon du Niger.

(C) 2012 International Formulae Group. All rights reserved.

Mots clés: Oignon, diversité, caractérisation agro-morphologique, Niger.

\section{INTRODUCTION}

L'oignon (Allium cepa L.) est originaire de la partie sud-ouest de l'Asie centrale (Afghanistan, Iran, et Pakistan) et les républiques méridionales de l'ancienne URSS (Jones et Mann, 1963 cité par De Bon, 1987).
L'espèce A. cepa renferme trois groupes, à savoir les oignons communs, les oignons $A$. aggregattum et les oignons $A$. prolilum, tous diploides $\quad(2 \mathrm{n}=2 \mathrm{x}=16)$. Il occupe le $4^{\text {ème }}$ rang mondial des légumes consommés avec une production annuelle mondiale de 25

() 2012 International Formulae Group. All rights reserved. DOI : http://dx.doi.org/10.4314/ijbcs.v6i6.11 
millions de tonnes après la tomate, le chou, et la pastèque (Silué et al ., 2010). L'oignon a été introduit au Niger à partir de l'Egypte par le circuit du commerce transsaharien qui était le moyen de contact et d'échange entre les pays de l'Afrique du Nord et ceux de l'Afrique noire (Assane, 2006). La superficie exploitée est aujourd'hui estimée à environ 30215 ha avec une production nationale annuelle de 560782 tonnes et une valeur monétaire d'environ 19 milliards de FCFA (RGCA, 2008). Il est cultivé dans toutes les régions du Niger sur des superficies variables : les principales zones de production se situent autour de la Maggia et de la vallée de la Tarka dans la région de Tahoua, autour du Goulbi à Maradi, dans l'Air à Agadez, autour de la Komadougou dans la région de Diffa, les Dallols Maouri et Bosso dans la région de Dosso et la Korama à Zinder. Les travaux de sélection conduits sur les écotypes collectés dans les années 60-70 ont permis de sélectionner trois variétés à savoir le Violet de Galmi, le Blanc de Galmi et le Blanc de Soumarana (Nabos, 1976). L'une de ces variétés nigériennes à savoir le Violet de Galmi est la plus prisé de la sous-région grâce à sa qualité condimentaire, sa coloration homogène, et son aptitude à la conservation. Afin de constituer une banque de gène qui contribuera à l'amélioration génétique de cette espèce, une collecte a été effectuée dans toutes les régions du Niger en 2008 et a permis de recenser 21 variétés et/ou écotypes locaux. Le matériel génétique collecté a fait l'objet de caractérisation morphologique à la station expérimentale de l'Institut National de la Recherche Agronomique du Niger (INRAN) à Konni (sud-est du Niger) afin de faire un regroupement et déterminer le nombre de variétés et/ou écotypes potentiellement existants au Niger.

\section{MATERIEL ET METHODES Site d'étude}

Les essais ont été conduits sur deux campagnes consécutives (2009 et 2010) à la station de recherche de l'INRAN à Konni, à $475 \mathrm{~km}$ à l'Est de Niamey.

Elle est comprise entre $13^{\circ} 81^{\prime} 92^{\prime \prime}$ de latitude Nord et $5^{\circ} 28^{\prime} 85^{\prime \prime}$ de longitude Est. Le climat de type sahélo-soudanien est caractérisé par une longue saison sèche (saison sèche froide et saison sèche chaude) qui va d'octobre à mai et une courte saison de pluies qui va de juin à septembre. La pluviométrie moyenne annuelle est d'environ $520 \mathrm{~mm}$. Les températures varient de $12{ }^{\circ} \mathrm{C}$ à $44{ }^{\circ} \mathrm{C}$ au cours de l'année. La texture du sol est à prédominance sablonneuse. C'est une des principales zones de production agricole par excellence au Niger (Assane, 2006).

\section{Matériel végétal \\ Le Tableau 1 présente les variétés et/ou écotypes évalués. \\ Mise en place de la culture et entretiens culturaux}

Les pépinières ont été mises en place durant les mois de novembre dans des planches de $1 \mathrm{~m}^{2}$ de superficie selon un semis en ligne. Comme fumure de fond, une dose de $1 \mathrm{~kg}$ de fumier organique a été apportée par planche. La fréquence d'arrosage a été de deux irrigations par jour et le désherbage est fait à la demande.

Des plants âgés de 45 jours ont été repiqués dans un dispositif en blocs complets randomisés à trois répétitions sur des parcelles élémentaires de $4 \mathrm{~m}^{2}$.

Après le repiquage, l'irrigation par submersion a été maintenue avec une fréquence de deux séances par semaine jusqu'au début de la bulbaison, puis ramenée à une séance par semaine jusqu'à la période de maturité. Le désherbage a été effectué à la demande.

La fumure d'entretien a été faite à raison de $10 \mathrm{~g} / \mathrm{m}^{2}$ de 15-15-15 deux semaines après repiquage et $10 \mathrm{~g} / \mathrm{m}^{2}$ à 75 jours après le repiquage. Au plan pathologique, un suivi régulier des plants a été fait de la pépinière à la récolte. 
Tableau 1: Les différentes variétés et/ou écotypes d'oignon du Niger et leurs origines.

\begin{tabular}{|c|c|c|c|c|c|}
\hline Ecotypes & Abréviation & Origine & Variété & Abréviation & Origine \\
\hline Blanc de Diffa & $\mathrm{BDa}$ & Diffa & Ayérou & Ay & Ayérou \\
\hline Blanc de Gotheye & BGo & Gotheye & Blanc de Soumarana & $\mathrm{BSa}$ & Soumarana \\
\hline Albassa Guidan Idder & AGI & G Idder & Ja Albassa & $\mathrm{JaA}$ & Soumarana \\
\hline Rose de Diffa & $\mathrm{RsDa}$ & Diffa & Blanc de skoukoutan & BSn & Matankari \\
\hline Rouge de Gaidam & $\mathrm{RgGa}$ & Diffa & Youri & YI & Loubiré \\
\hline Violet de Galmi Diffa & VGDa & Diffa & Violet de galmi Gaya & VGGa & Gaya \\
\hline Yaouri Izé & YaI & Gaya & Violet de Galmi Ayérou & VGAy & Ayérou \\
\hline Albassa Madarounfa & AMfa & Madarounfa & Jan Iri & JaI & Sabon Guida \\
\hline Guidan Magagi & GM & G.magagi & Kankaré & $\mathrm{Ke}$ & G Idder \\
\hline $\begin{array}{l}\text { Madarounfa Dan Radi } \\
\text { Tawraka }\end{array}$ & $\begin{array}{c}\text { MfaDR } \\
\text { Twa }\end{array}$ & $\begin{array}{l}\text { Madarounfa } \\
\text { Galmi }\end{array}$ & Tassa Galmi & $\mathrm{TaG}$ & G Idder \\
\hline
\end{tabular}

\section{Observations et mesures}

Les observations et mesures ont porté sur la longueur, la largeur, le poids, la couleur, et la densité des feuilles, le port, et la vigueur des plants, les poids frais et sec, le diamètre, la hauteur, la couleur et l'uniformité des bulbes, la couleur de la chair et l'épaisseur des écailles au niveau des bulbes et le nombre de bourgeons végétatifs. La teneur en matière sèche a été déduite selon la formule suivante :

Teneur ne matière sèche $($ TMS $)(\%)=$ (Poids sec / Poids frais) x 100.

\section{Traitement des données}

Les données collectées sur 2 ans ont été traitées à l'aide du logiciel STATIX 8 pour l'analyse de variance (ANOVA) et XLSTAT 2012.6.03 pour l'analyse à composante principale(ACP).

\section{RESULTATS ET DISCUSSION}

Les résultats des observations et mesures faites durant toute la durée du cycle productif de la culture sont résumés dans les Tableaux 2 et 3 .

Le diamètre des bulbes varie entre 6,19 $\mathrm{cm}$ (écotype Rose de Diffa) et $8,56 \mathrm{~cm}$ (Kankaré).

Quant à la hauteur des bulbes, la plus petite, qui est de $3,99 \mathrm{~cm}$, a été observée chez l'écotype Albassa Guidan Idder et la plus élevée chez Kankaré avec $8,79 \mathrm{~cm}$.
Pour ce qui est de la longueur des feuilles, elle varie de $17,03 \mathrm{~cm}$ à $41 \mathrm{~cm}$. Les feuilles les plus longues ont été enregistrées au niveau de l'écotype Blanc de Soumarana, et l'écotype Jan Iri présente les feuilles les plus petites. Le taux de matière sèche (MS) des feuilles et des bulbes a aussi été déterminé. Au niveau des bulbes, les écotypes Blanc de Gotheye $(13,24 \%)$ et Blanc de Diffa $(12,74 \%)$ ont les valeurs moyennes de MS les plus élevées. Ils sont suivis respectivement par Yaouri Ize puis Madarounfa Dan Radi avec respectivement $12,46 \%$ et $12,42 \%$ de MS. Les autres écotypes ont les valeurs comprises entre $10 \%$ et $11 \%$ en moyenne de MS. L'écotype Blanc de Soukoukoutan présente le plus petit taux de matière sèche, soit $8,91 \%$. Au niveau des feuilles, le plus fort taux de matière sèche a été observé chez l'écotype Tawraka.

Concernant les bourgeons végétatifs, ils sont plus nombreux au niveau de l'écotype Rose de Diffa qui en présente une moyenne de 10 par bulbe tandis que le Violet de Galmi collecté à Diffa et à Ayérou présente à peine 2 bourgeons végétatifs. Quant au rendement en bulbes, il varie selon les écotypes. Le plus haut rendement est observé chez l'écotype Guidan Magagi avec 34,22 t/ha et le plus bas chez l'écotype Rose de Diffa (17,75 t/ha). Il faut noter que ce dernier écotype est le seul à présenter un rendement inférieur à la moyenne 
nationale en milieu paysan qui est de 18,5 t/ha (RCGA, 2008).

Ces caractères quantitatifs et qualitatifs des variétés et/ou écotypes d'oignon étudiés sont ceux recommandés par IPGRI (2001) pour la caractérisation agro-morphologique de cette espèce. Ces mêmes recommandations ont été utilisées dans les programmes de sélection, telles que les études d'adaptabilité de l'oignon en Côte d'Ivoire présentées par Silué en 2003 et au Burkina Faso par Rouamba en 2001. Aussi, plusieurs de ces caractères ont permis de faire des choix des écotypes de Niébé pour des utilisations aux fins de production ou de la recherche au Burkina Faso (Ouedraougo et al., 2010). En effet, les caractères discriminants peuvent être uniquement quantitatifs ou qualitatifs ou la combinaison des deux.

L'analyse en composantes principales (ACP) a montré qu'il y a une corrélation positive entre la longueur des feuilles et la hauteur des bulbes $\left(r^{2}=0,704\right)$, entre le diamètre des bulbes et le rendement $\left(r^{2}=\right.$ $0,653)$, entre la hauteur de bulbes et leur diamètre $\left(\mathrm{r}^{2}=0,618\right)$, entre le nombre de bourgeons végétatifs et la matière sèche des bulbes $\left(r^{2}=0,558\right)$, et enfin entre le cycle et la longueur des feuilles $\left(r^{2}=0,522\right)$ (Tableau 4).

En outre, ces résultats montrent que les deux premiers axes ont exprimé la plus grande variabilité (59,02\%). La contribution de chaque caractère discriminant est notée dans le Tableau 5. L'axe 1 qui donne $36,79 \%$ d'informations est défini par les caractères végétatifs qui sont le diamètre, la hauteur des bulbes et la longueur des feuilles. L'axe 2 défini avec $22,23 \%$ de contribution, donne les informations sur le rendement et la matière sèche des feuilles (Figure 1).

Pour le regroupement des écotypes à partir des caractères observés, l'analyse est basée sur la distance euclidienne. Ainsi, trois groupes se sont distingués (Figure 2) :

- Le groupe 1 contient quinze écotypes (71\%) de couleurs différentes qui sont entre autres la couleur blanche chez les écotypes Blanc de Soumarana et Blanc de Gothey; la couleur rouge chez Yaourizé et Jan Iri; la couleur rose chez la Rose de Diffa et le violet chez les dix autres écotypes.

Il en est de même pour le groupe 3, composé de deux écotypes $(10 \%)$ qui sont le Rouge de Gaidam et Soukoukoutan de couleurs différentes.

- Le groupe 2 comprend quatre écotypes (19\%) à savoir Tassa Galmi, Guidan Magagi, Tawraka tous de couleur violette. On constate en plus que les écotypes à hauts rendements sont en majorité originaires de la Maggia (région de Tahoua). Ce sont entre autres les écotypes Tawraka (32,21 t/ha), Guidan Magagi (34,22 t/ha), Tassa Galmi $(29,69 \mathrm{t} / \mathrm{ha})$. Les autres écotypes à hauts rendements sont originaires de la vallée du Goulbi de Maradi : Albassa Madarounfa (30,66 t/ha), Madarounfa Dan Radi (27,32 t/ha).

Il faut noter que la variabilité est beaucoup plus observée à l'intérieur des groupes $(64,18 \%)$ qu'entre les groupes $(35,82 \%)$. En effet, l'analyse factorielle discriminante a montré que le groupe 2 est non seulement lié au groupe 3 par le rendement $(21,82 \mathrm{t} / \mathrm{ha}$ en moyenne, pour le groupe 2 et 21,98 t/ha pour le groupe 3 ) mais aussi au groupe 1 par le cycle (149 jours pour le groupe 1 et 152 jours en moyenne pour le groupe 2) et par le nombre de bourgeons végétatifs avec une moyenne de 5 pour les deux groupes.

L'analyse de variance a révélé une différence significative entre le diamètre des bulbes ( $\mathrm{p}<0,03$ ), la longueur des feuilles ( $\mathrm{p}<$ 0,002), la hauteur des bulbes, le nombre de bourgeons végétatifs et la matière sèche des feuilles ( $\mathrm{p}<0,000)$. Par contre, l'analyse factorielle par correspondance (AFC) n'a montré aucune corrélation au niveau des caractères qualitatifs. 
Tableau 2: Les caractéristiques agro-morphologiques quantitatives des variétés et/ou écotypes d'oignon évalués.

\begin{tabular}{lcccccccc}
\hline Ecotypes & $\begin{array}{c}\text { Rdt } \\
(\mathbf{t} / \mathbf{h a})\end{array}$ & $\begin{array}{c}\text { Diab } \\
(\mathbf{c m})\end{array}$ & $\begin{array}{c}\text { Hautb } \\
(\mathbf{c m})\end{array}$ & $\begin{array}{c}\text { Longf } \\
(\mathbf{c m})\end{array}$ & $\begin{array}{c}\text { Msf } \\
(\boldsymbol{\%})\end{array}$ & $\begin{array}{c}\text { Msb } \\
(\boldsymbol{\%})\end{array}$ & Bv & Cycle \\
\hline BDA & 23,67 & 6,82 & 5,26 & 29,46 & 12,67 & 12,74 & 4,00 & 140,00 \\
BGo & 14,89 & 5,86 & 4,74 & 25,69 & 10,17 & 13,42 & 7,00 & 140,00 \\
TaG & 29,69 & 8,52 & 8,47 & 31,89 & 11,89 & 10,00 & 5,00 & 150,00 \\
RsDa & 17,75 & 6,19 & 4,58 & 32,02 & 12,59 & 12,10 & 10,00 & 180,00 \\
RgGa & 24,69 & 7,41 & 5,51 & 26,61 & 8,06 & 9,45 & 4,00 & 180,00 \\
VGDa & 18,32 & 7,27 & 4,38 & 21,39 & 10,82 & 11,10 & 2,00 & 150,00 \\
Yal & 21,75 & 7,12 & 4,56 & 20,60 & 12,19 & 12,46 & 6,00 & 140,00 \\
MfaDR & 27,32 & 7,07 & 5,84 & 29,07 & 9,08 & 12,42 & 6,00 & 160,00 \\
GM & 34,22 & 8,06 & 6,08 & 20,32 & 11,71 & 9,39 & 3,00 & 150,00 \\
Amfa & 30,66 & 7,54 & 5,42 & 19,74 & 10,47 & 11,47 & 4,00 & 160,00 \\
Twa & 32,21 & 8,34 & 6,00 & 24,28 & 13,92 & 11,06 & 6,00 & 150,00 \\
Ay & 26,62 & 8,19 & 4,83 & 25,57 & 9,94 & 11,24 & 3,00 & 140,00 \\
Bsa & 24,18 & 6,70 & 5,18 & 27,19 & 12,10 & 11,44 & 4,00 & 160,00 \\
JaA & 21,33 & 6,80 & 4,85 & 17,31 & 12,61 & 12,01 & 7,00 & 150,00 \\
BSn & 19,29 & 7,80 & 8,37 & 41,00 & 8,04 & 9,91 & 1,00 & 180,00 \\
VGGa & 19,81 & 7,50 & 3,97 & 23,95 & 9,85 & 10,58 & 2,00 & 140,00 \\
YI & 20,98 & 6,64 & 5,96 & 25,81 & 9,54 & 12,24 & 3,00 & 140,00 \\
Ke & 23,15 & 8,56 & 8,79 & 38,09 & 10,86 & 11,00 & 5,00 & 160,00 \\
AGI & 17,81 & 6,49 & 3,99 & 23,81 & 9,50 & 10,29 & 3,00 & 150,00 \\
Jal & 21,84 & 6,53 & 4,41 & 17,08 & 12,78 & 10,11 & 7,00 & 140,00 \\
VGAy & 20,49 & 7,58 & 4,38 & 26,33 & 10,07 & 10,92 & 2,00 & 140,00 \\
\hline
\end{tabular}

Blanc de Diffa (BDA), Blanc de Gotheye (BGo), Tassa Galmi (TaG), Rose de Diffa (RsDa) ; Rouge de Gaidam (RgGa) ; Violet de Galmi Diffa (VGDa), Yaourizé (YaI); Madarounfa Dan Radi (MfaDR); Guidan Magagi (GM); Albassa Madarounfa (AMfa); Tawraka (Twa); Ayérou (Ay); Blanc de Soumarana (BSa); Ja Albassa (JaA); Blanc de Soukoukoutan (BSn); Violet de Galmi Gaya (VGGa); Youri (YI) ; Kankaré (Ke) ; Jan Iri (JaI) ; Albassa Guidan Idder (AGI) ; Violet de Galmi Ayérou (VGAy); Rendement (Rdt) ; Diamètre des bulbes (Diab) ; Hauteur des bulbes (Hautb) ; Longueur des feuilles (Longf); Matière sèche dans les feuilles (Msf); Matière sèche dans les bulbes (Msb) ; Bourgeons végétatifs $(\mathrm{Bv})$.

Tableau 3: Les caractéristiques agro-morphologiques qualitatives des variétés et/ou écotypes d'oignon évalués.

\begin{tabular}{|c|c|c|c|c|c|c|c|c|}
\hline Ecotypes & $\begin{array}{l}\text { vigueur } \\
\text { des } \\
\text { plants }\end{array}$ & $\begin{array}{c}\text { Couleur } \\
\text { des } \\
\text { feuilles }\end{array}$ & $\begin{array}{c}\begin{array}{c}\text { Densité } \\
\text { des } \\
\text { feuilles }\end{array}\end{array}$ & Port & $\begin{array}{l}\text { Uniformit } \\
\text { é des } \\
\text { bulbes }\end{array}$ & $\begin{array}{c}\text { Couleur } \\
\text { bulbe }\end{array}$ & $\begin{array}{c}\text { Couleur } \\
\text { chair }\end{array}$ & $\begin{array}{c}\text { Epaisseu } \\
\text { r des } \\
\text { écailles }\end{array}$ \\
\hline BDa & bonne & Vert-gris & faible & érigé & forte & $\begin{array}{l}\text { Rose } \\
\text { clair }\end{array}$ & $\begin{array}{l}\text { Rose } \\
\text { clair }\end{array}$ & Moyenne \\
\hline BGo & faible & Vert-gris & élevée & érigé & moyenne & blanc & blanc & Moyenne \\
\hline AGI & moyenne & Vert-gris & moyenne & érigé & moyenne & violet & violet & Moyenne \\
\hline RsDa & bonne & vert & moyenne & érigé & moyenne & rose & violet & Moyenne \\
\hline RgGa & bonne & vert & élevée & érigé & moyenne & violette & $\begin{array}{c}\text { Blanc } \\
\text { sale }\end{array}$ & Moyenne \\
\hline VGDa & bonne & vert-gris & moyenne & érigé & moyenne & violette & $\begin{array}{c}\text { Blanc } \\
\text { sale }\end{array}$ & Moyenne \\
\hline
\end{tabular}




\begin{tabular}{|c|c|c|c|c|c|c|c|c|}
\hline YaI & moyenne & Vert-gris & moyenne & érigé & forte & rouge & violet & Moyenne \\
\hline AMfa & bonne & vert & moyenne & érigé & moyenne & Violette & $\begin{array}{c}\text { Blanc } \\
\text { sale }\end{array}$ & Moyenne \\
\hline GM & moyenne & Vert-gris & moyenne & érigé & moyenne & violette & $\begin{array}{c}\text { Blanc } \\
\text { sale }\end{array}$ & Moyenne \\
\hline MfaDR & moyenne & Vert-gris & moyenne & érigé & moyenne & violette & $\begin{array}{c}\text { Blanc } \\
\text { sale }\end{array}$ & Moyenne \\
\hline Twa & moyenne & vert-gris & faible & érigé & moyenne & violette & violet & Mince \\
\hline Ay & moyenne & vert-gris & moyenne & érigé & moyenne & violette & $\begin{array}{c}\text { Blanc } \\
\text { sale }\end{array}$ & Mince \\
\hline BSa & bonne & Vert-gris & moyenne & érigé & forte & blanche & Blanc & Mince \\
\hline $\mathbf{J a A}$ & moyenne & Vert-gris & élevée & érigé & moyenne & violette & $\begin{array}{l}\text { Violet } \\
\text { clair }\end{array}$ & Moyenne \\
\hline BSn & bonne & Vert-gris & moyenne & érigé & moyenne & blanche & $\begin{array}{c}\text { Blanc } \\
\text { sale }\end{array}$ & Moyenne \\
\hline YI & bonne & Vert-gris & moyenne & érigé & faible & violette & violet & Epaisse \\
\hline VGGa & moyenne & vert-gris & moyenne & érigé & moyenne & violette & blanc & Moyenne \\
\hline VGAy & moyenne & Vert-gris & moyenne & érigé & moyenne & violette & $\begin{array}{l}\text { Violet } \\
\text { clair }\end{array}$ & Epaissse \\
\hline JaI & bonne & vert & élevée & érigé & moyenne & violette & $\begin{array}{l}\text { Violet } \\
\text { clair }\end{array}$ & Moyenne \\
\hline $\mathbf{K e}$ & moyenne & vert-gris & moyenne & érigé & moyenne & violette & $\begin{array}{l}\text { Violet } \\
\text { clair }\end{array}$ & Mince \\
\hline TaG & moyenne & Vert-gris & moyenne & érigé & moyenne & violette & $\begin{array}{l}\text { Violet } \\
\text { clair }\end{array}$ & Moyenne \\
\hline
\end{tabular}

Blanc de Diffa (BDA), Blanc de Gotheye (BGo), Tassa Galmi(TaG), Rose de Diffa (RsDa) ; Rouge de Gaidam (RgGa) ; Violet de Galmi Diffa (VGDa), Yaourizé (YaI); Madarounfa Dan Radi (MfaDR); Guidan Magagi (GM); Albassa Madarounfa (AMfa); Tawraka (Twa); Ayérou (Ay); Blanc de Soumarana (BSa); Ja Albassa (JaA); Blanc de Soukoukoutan (BSn); Violet de Galmi Gaya (VGGa); Youri (YI) ; Kankaré (Ke) ; Jan Iri (JaI) ; Albassa Guidan Idder (AGI) ; Violet de Galmi Ayérou (VGAy).

Tableau 4: Matrice de corrélation des caractères quantitatifs.

\begin{tabular}{ccccccccc}
\hline Variables & Rdt & Diab & Hautb & Longf & Msf & Msb & Bv & Cycle \\
\hline Rdt & $\mathbf{1}$ & & & & & & & \\
Diab & $\mathbf{0 , 6 5 3}$ & $\mathbf{1}$ & & & & & & \\
Hautb & 0,362 & $\mathbf{0 , 6 1 8}$ & $\mathbf{1}$ & & & & & \\
Longf & $-0,134$ & 0,280 & $\mathbf{0 , 7 0 4}$ & $\mathbf{1}$ & & & & \\
Msf & 0,260 & $-0,027$ & $-0,118$ & $-0,333$ & $\mathbf{1}$ & & & \\
Msb & $-0,319$ & $-0,531$ & $-0,280$ & $-0,056$ & 0,222 & $\mathbf{1}$ & & \\
Bv & $-0,054$ & $-0,397$ & $-0,110$ & $-0,119$ & $\mathbf{0 , 5 5 8}$ & 0,442 & $\mathbf{1}$ & \\
Cycle & 0,026 & 0,067 & 0,383 & $\mathbf{0 , 5 2 2}$ & $-0,293$ & $-0,291$ & 0,126 & $\mathbf{1}$ \\
\hline
\end{tabular}

Rendement (Rdt) ; Diamètre des bulbes (Diab) ; Hauteur des bulbes (Hautb); Longueur des feuilles (Longf) ; Matière sèche dans les feuilles $(\mathrm{Msf})$; Matière sèche dans les bulbes $(\mathrm{Msb})$; Bourgeons végétatifs $(\mathrm{Bv})$. 
Tableau 5: La contribution de chaque caractère étudié des 21écotypes.

\begin{tabular}{lccccc}
\hline & F1 & F2 & F3 & F4 & F5 \\
\hline Rdt & 0,450 & $\mathbf{0 , 7 6 3}$ & 0,159 & 0,092 & 0,393 \\
Diab & $\mathbf{0 , 7 9 9}$ & 0,478 & $-0,026$ & $-0,178$ & $-0,033$ \\
Hautb & $\mathbf{0 , 8 0 6}$ & $-0,023$ & 0,419 & $-0,274$ & $-0,048$ \\
Longf & $\mathbf{0 , 6 2 4}$ & $-0,554$ & 0,371 & $-0,307$ & $-0,125$ \\
Msf & $-0,395$ & $\mathbf{0 , 6 2 9}$ & $\mathbf{0 , 5 2 2}$ & $-0,023$ & $-0,342$ \\
Msb & $-0,656$ & $-0,210$ & 0,338 & $-0,510$ & 0,356 \\
Bv & $-0,491$ & 0,047 & $\mathbf{0 , 7 8 9}$ & 0,213 & 0,007 \\
Cycle & 0,486 & $-0,468$ & 0,401 & $\mathbf{0 , 5 6 5}$ & 0,139 \\
Valeur propre & 2,943 & 1,778 & 1,510 & 0,835 & 0,437 \\
Variabilité $(\%)$ & 36,794 & 22,231 & 18,875 & 10,441 & 5,462 \\
\% cumulé & 36,794 & 59,024 & 77,899 & 88,341 & 93,803 \\
\hline
\end{tabular}

Rendement (Rdt); Diamètre des bulbes (Diab); Hauteur des bulbes (Hautb); Longueur des feuilles (Longf); Matière sèche dans les feuilles (Msf); Matière sèche dans les bulbes (Msb); Bourgeons végétatifs (Bv).

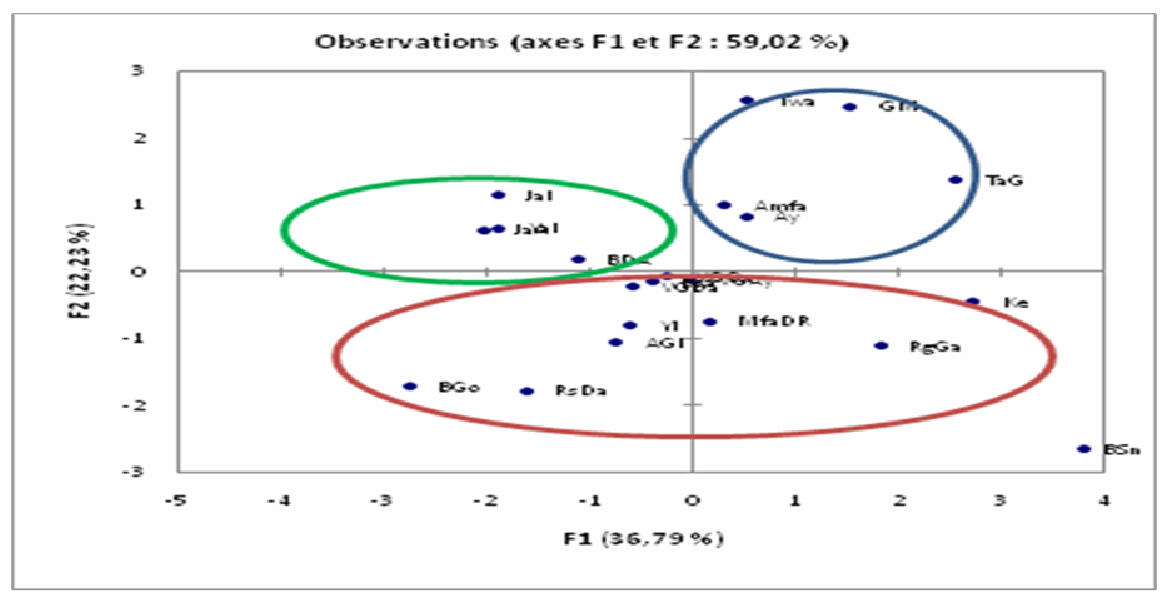

Figure 1: Distribution des différents écotypes par rapport aux principaux axes. 


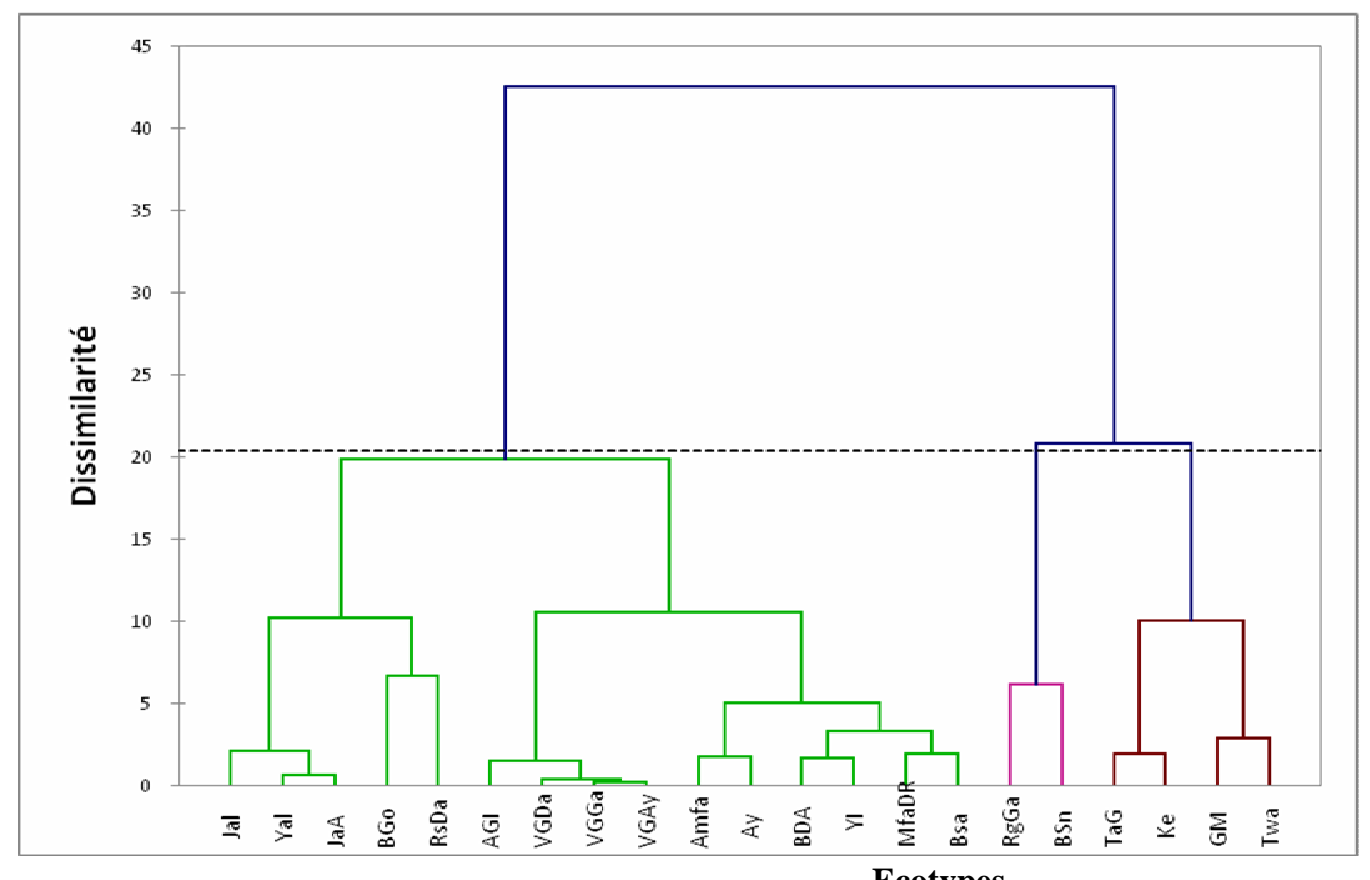

Ecotypes

Figure 2: Dendrogramme identifiant trois groupes homogènes.

Les couleurs des bulbes observées sont multiples. Elles varient du rouge, violet, violet clair, rose au blanc. Ces bulbes présentent une chair de couleur rose clair, blanc sale, violette avec des écailles minces ou moyennes. L'uniformité est forte ou moyenne sauf chez l'écotype Youri où elle est faible. Les écotypes sont tous de port érigé avec une vigueur bonne à moyenne pour la majorité. La faible vigueur a été observée uniquement chez l'écotype Blanc de Gotheye. Les feuilles sont de couleur verte ou gris-vert avec une densité élevée, moyenne, et faible surtout chez l'écotype Rose de Diffa et Tawraka.

Issues des différentes zones agroclimatiques et cultivées sur différents types de sols, ces écotypes sont donc liés par les caractères végétatifs discriminants cités plus haut. Des études similaires ont permis de caractériser 34 variétés de riz (Moukoumbi et al., 2011), les classant dans 3 groupes différents à partir de 11 caractères quantitatifs des 23 caractères étudiés. En Côte d'Ivoire, l'oseille de Guinée (Hibiscus sabdariffa) a été caractérisée et classée selon les caractères quantitatifs et qualitatifs (Sié et al., 2009) à Khorogho. Il en est de même pour la caractérisation des accessions du mil dans la même région de Khorogho à partir de 11 caractères quantitatifs qui ont permis le rapprochement des accessions aux deux groupes de mil de l'Afrique de l'Ouest: groupe tardif et groupe précoce (Akanvou et al., 2012). Aussi, les études menées par Bakasso (2010) au Niger ont défini deux groupes différents d'oseille de Guinée pour les caractères agronomiques qui sont le cycle de floraison, le poids des cent graines et le poids du calice.

\section{Conclusion}

A l'issue de cette étude, une grande diversité agro-morphologique et physiologique a été observée chez les variétés et/ou écotypes locaux d'oignons collectés dans différentes régions du Niger. L'analyse des caractères agro-morphologiques et physiologiques quantitatifs a permis de distinguer trois groupes dont $71 \%$ de variétés 
et/ou écotypes locaux pour le premier, $19 \%$ pour le deuxième et $10 \%$ pour le troisième, tandis qu'aucune corrélation n'a été révélée au niveau des caractères qualitatifs. La variabilité observée dans les groupes est plus grande que celle qui existe entre les groupes, ce qui explique l'appartenance des écotypes de différentes couleurs et de différentes régions dans un même groupe. Un marquage moléculaire s'avère nécessaire pour confirmer cette diversité des variétés et/ou écotypes locaux d'oignons du Niger.

\section{REFERENCES}

Akanvou L. 2012. Evaluation de la Diversité agro-morphologique des accessions de mil (Pennisetum glaucum) (L) R.Br) collectées en Côte d'Ivoire. J. Applied Biosciences, 50: 3468-3477.

Assane DM. 2006. Les effets de le réappropriation de la culture du « violet de Galmi » par les producteurs de la région de Tahoua-NIGER sur la dynamique du territoire local, l'organisation social et économique. Thèse de Doctorat, Université de Toulouse-Le Mirail, p. 281.

Bakasso Y. 2010. Ressources génétiques des Roselles (Hibiscus sabdarifa L.) au Niger: évaluation agro-morphologique et génétique. Thèse de Doctorat d'Etat, Université Abdou Moumouni de Niamey, p. 139.

Hubert de Bon. 1987. Développement de l'oignon (Allium Cepa L) en zone Tropicale, Etude particulière de la variété Violet de Galmi, Thèse de doctorat, l'institut National Agronomique Paris-Grignon, p. 148.

IPGRI, ECP/GR, AVRDC. 2001. Descriptors for Allium (Allium spp). International Plant Genetic Ressources Institue:
Rome, Italy; European Cooperative Programme for Crop Genetic Ressources Networks (ECP/GR). Asian Vegetable Rsearch and Development Center: Taiwan.

Moukoumbi.YD. 2011. Assessiming phenotypic diversity of interspecific rice varieties using agro-mrophological characterization. Journal of Plant Breeding and Crop Science, 3(5): 74-86.

Nabos J. 1976. L'amélioration de l'oignon (Allium cepa L.) au Niger. Agronomie Tropicale, 31(4): 387-397.

Ouedrago JT, Sawadogo M, TIGNEGRE JB, DRABO I, Balma D. 2010. Caractérisation agro morphologique et moléculaire des cultivars locaux (Vigna unguiculata) du Burkina Faso. Cameroon Journal of Experimental Biology, 6(1): 31-40.

RGAC. 2008. République du Niger, Ministère $\mathrm{du}$ Développement Agricole, Recensement Général de l'Agriculture et du Cheptel.

Rouamba Ghene RH, Bâ D, Ricroch A, Currah L. 2001. Agronomic and physiological evaluation of some régional populations of onion in field and storage trials in West Africa. Trop. Sciences, 41: 78-84.

Sié RS, Akaffou DS, Séka D, Konan K JL, Toueix Y, Charles G, Djè Y, Branchard M. 2009. Caractérisation de la diversité et de l'évaluation agro morphologique d'une collection d'Hibiscus Sabdariffa $L$ en Côte d'Ivoire. Afrique Science, 5(3): 65-76.

Silué S, Fondjio L, Coulibaly MY, Magein H. 2003. Sélection des variétés d'oignon (Allium Cepa L) adaptées au Noud de la Côte d'Ivoire. Tropicultura, 21(3): 129134. 